\title{
A Case of Intracranial Hypotension after Horse Riding
}

\author{
Sibel Karsidag ${ }^{a}$ \\ Nilgun Cinar $^{\mathrm{a}}$ \\ Sevki Sahin ${ }^{\mathrm{a}}$ \\ Miruna Florentina Ates ${ }^{a}$ \\ Necati Alp Tabak ${ }^{\mathrm{b}}$ \\ a'Department of Neurology, \\ Faculty of Medicine Maltepe University, \\ Istanbul, Turkey \\ bDepartment of Radiology, \\ NPIstanbul Brain Hospital, Istanbul, \\ Turkey
}

Dear Editor,

Trauma is the cause of $80-90 \%$ cases of cerebrospinal fluid (CSF) leaks. ${ }^{1}$ CSF leakage may be early onset, or can be delayed for more than 1 week after trauma. Spinal sequelae occur as a complication to CSF leaks. ${ }^{1,2}$ CSF leakage and intracranial hypotension (IH) have been rarely reported after vigorous sports activities, with the literature including two cases that occurred after playing tennis and squash. ${ }^{3}$ Severe injuries secondary to falls and crush or kicking can occur during horse riding, ${ }^{4}$ but CSF leakage after horse riding has not been reported previously.

Here we describe a patient who experienced severe headache after horse riding. A 35-yearold male medical doctor without previously headache or trauma history was admitted 2 days after experiencing a severe headache following horse riding for 2 hours. He reported that this had been his longest experience of horse riding. His symptoms were characterized by an orthostatic headache that tended to improve in a lying position and worsen when sitting or standing. A neurologic examination revealed mild neck stiffness without any other sign of pyramidal irritation. His vital findings and blood chemistry parameters were normal. The findings of brain magnetic resonance imaging (MRI) were normal, but spinal MRI demonstrated epidural fluid collection starting from the T4 vertebra in T2-weighted images (Fig. $1 \mathrm{~A}$ and $\mathrm{B})$.

The patient was diagnosed with a headache related to IH due to CSF leakage. Because no other risk factor was identified and the clinical findings started after a long period of horse riding, we attributed the spontaneous $\mathrm{IH}$ to horse-riding-related minor trauma in the epidural area. The patient did not accept the epidural blood-patch procedure. He received bed rest, an intravenous balanced electrolyte solution at 2,000 mL/day, $400 \mathrm{mg} /$ day theophylline, and oral analgesic treatment that included $500 \mathrm{mg}$ of acetaminophen, $30 \mathrm{mg}$ of caffeine, and 10 $\mathrm{mg}$ of codeine given twice daily. Two weeks later his symptoms had partially improved. Follow-up MRI perform 1 month later showed regression of the fluid collection (Fig. 1C).

IH is usually a self-limiting disease caused by CSF leakage. ${ }^{5}$ It is reported that spinal epidural fluid collection has a sensitivity of $67-100 \%$ or diagnosing IH based on MRI findings. ${ }^{6}$ Although hydration and analgesic treatment is sufficient in most cases, the application of an intrathecal epidural blood patch may be necessary. ${ }^{5}$

To the best of our knowledge, this is the first case of IH related to CSF leakage caused by horse riding in the literature. Long periods of participation in vigorous sports activities can cause a transitory leakage of CSF and consequently IH.

\section{Conflicts of Interest}

The authors have no financial conflicts of interest.

\section{REFERENCES}

1. Yilmazlar S, Arslan E, Kocaeli H, Dogan S, Aksoy K, Korfali E, et al. Cerebrospinal fluid leakage complicating skull base fractures: analysis of 81 cases. Neurosurg Rev 2006;29:64-71.

@ This is an Open Access article distributed under the terms of the Creative Commons Attribution Non-Commercial License (https://creativecommons.org/licenses/by-nc/4.0) which permits unrestricted non-commercial use, distribution, and reproduction in any medium, provided the original work is properly cited. 

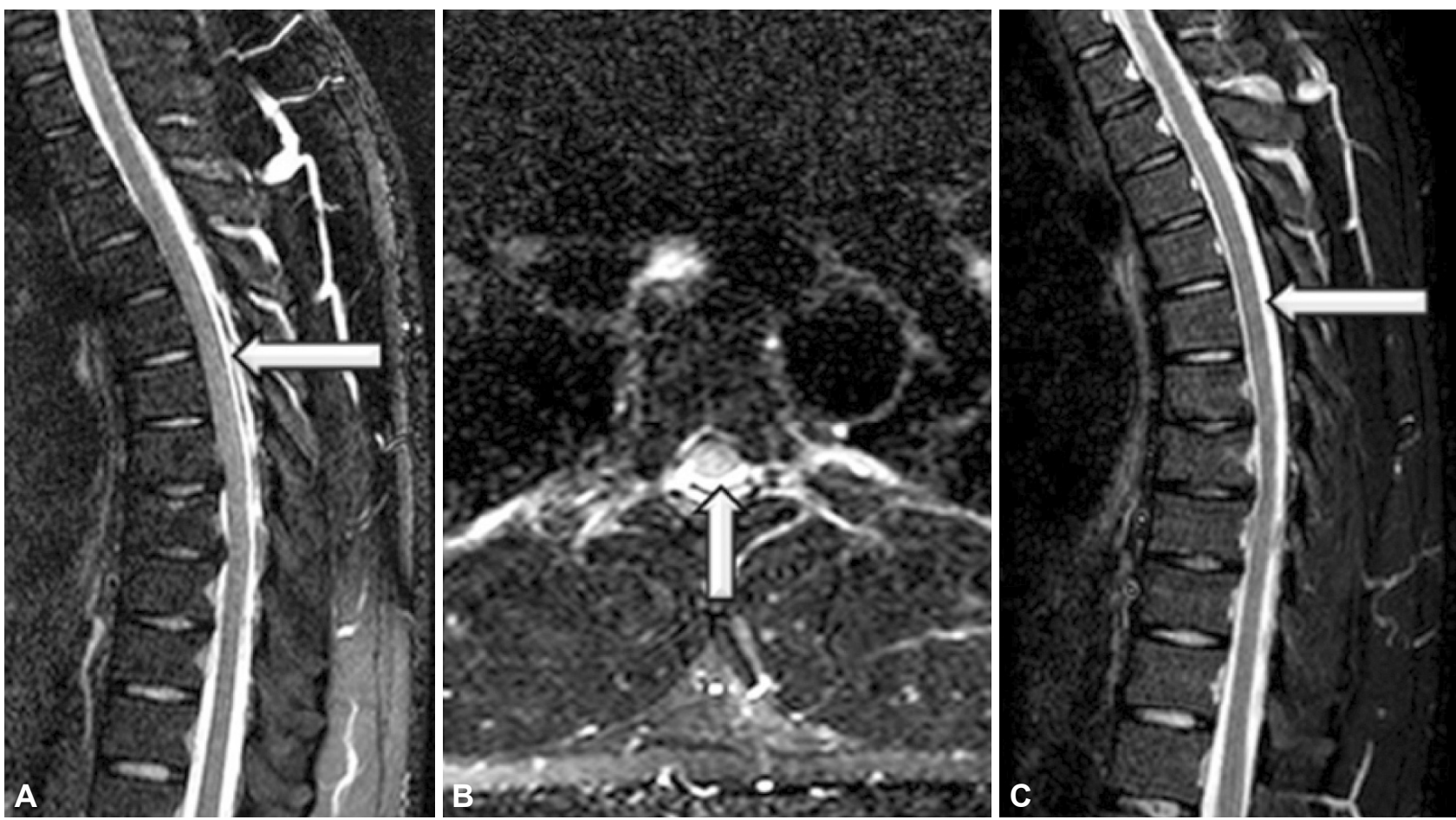

Fig. 1. Epidural cerebrospinal fluid collection. Arrows show (A) epidural fluid collection evident in a baseline MRI fat-saturated sagittal and (B) axial image. The dural sac is seen as a thin low-intensity ring of constant thickness. Epidural fluid is located dorsally. C: Arrow shows no fluid collection in the epidural space in follow-up MRI in a a fat-saturated sagittal image. MRI: magnetic resonance imaging.

2. Oh JW, Kim SH, Whang K. Traumatic cerebrospinal fluid leak: diagnosis and management. Korean J Neurotrauma 2017;13:63-67.

3. Garcia-Albea E, Cabrera F, Tejeiro J, Jimenez-Jimenez FJ, Vaquero A. Delayed postexertional headache, intracranial hypotension and racket sports. J Neurol Neurosurg Psychiatry 1992;55:975-980.

4. O'Connor S, Hitchens PL, Fortington LV. Hospital-treated injuries from horse riding in Victoria, Australia: time to refocus on injury prevention? BMJ Open Sport Exerc Med 2018;4:e000321.
5. Michali-Stolarska M, Bladowska J, Stolarski M, Sąsiadek MJ. Diagnostic imaging and clinical features of intracranial hypotension-review of literature. Pol J Radiol 2017;82:842-849.

6. Yagi T, Horikoshi T, Senbokuya N, Murayama H, Kinouchi H. Distribution patterns of spinal epidural fluid in patients with spontaneous intracranial hypotension syndrome. Neurol Med Chir (Tokyo) 2018;58: 212-218. 